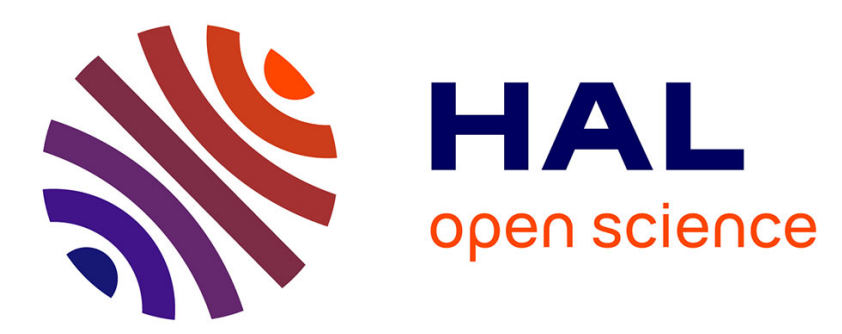

\title{
Misorientation dependence of the grain boundary migration rate: role of elastic anisotropy
}

Thiebaud Richeton, Xiaolei Chen, Stéphane Berbenni

\section{To cite this version:}

Thiebaud Richeton, Xiaolei Chen, Stéphane Berbenni. Misorientation dependence of the grain boundary migration rate: role of elastic anisotropy. Philosophical Magazine, 2020, 1000 (19), pp.2436-2457. 10.1080/14786435.2020.1779368 . hal-02929635

\section{HAL Id: hal-02929635 \\ https://hal.univ-lorraine.fr/hal-02929635}

Submitted on 3 Sep 2020

HAL is a multi-disciplinary open access archive for the deposit and dissemination of scientific research documents, whether they are published or not. The documents may come from teaching and research institutions in France or abroad, or from public or private research centers.
L'archive ouverte pluridisciplinaire HAL, est destinée au dépôt et à la diffusion de documents scientifiques de niveau recherche, publiés ou non, émanant des établissements d'enseignement et de recherche français ou étrangers, des laboratoires publics ou privés. 


\title{
Misorientation dependence of the grain boundary migration rate: role of elastic anisotropy
}

\author{
T. Richeton ${ }^{\mathrm{a}}$ and X. Chen ${ }^{\mathrm{a}}$ and S. Berbenni ${ }^{\mathrm{a}}$ \\ aUniversité de Lorraine, CNRS, Arts et Métiers ParisTech, LEM3, F-57000 Metz, France
}

\begin{abstract}
ARTICLE HISTORY
Compiled September 3, 2020

ABSTRACT

In this work, numerical computations of image forces and stored energy during the growth of a strain free grain within a recovered matrix containing an array of identical dislocations are performed. A decrease of the stored energy is observed when image forces are attractive and an increase when image forces are repulsive. The variation of elastic energy depends closely on the material elastic anisotropy, the number of dislocations in the array and its initial distance to grain boundary. For an array of 20 edge dislocations and the motion of a planar grain boundary over approximately 180 atomic spacing, the magnitude of the energy variation is on the order of one dislocation line energy for $\mathrm{Al}$ and six for $\mathrm{Cu}$, which is significant in both cases. Furthermore, by sweeping the whole orientation space for FCC crystals, it is shown that grain boundaries near a $\langle 111\rangle 40^{\circ}$ misorientation are among those that can display the highest attractive forces on edge dislocations. Since grain boundary migration is driven by the reduction of stored energy, the results are analyzed in light of experimental recrystallization data in FCC metals which show that the fastest moving boundaries are often characterized by misorientation axis around $\langle 111\rangle$ and misorientation angle close to $40^{\circ}$.
\end{abstract}

\section{KEYWORDS}

grain boundaries; image forces; dislocations; recrystallization; anisotropic elasticity; Stroh formalism

\section{Introduction}

During static recrystallization, some grain boundaries $(\mathrm{GBs})$ are moving in order to reduce the stored energy of the material. In addition to the effects of GB curvature [1], surface tension [1], temperature [2, 3], impurity concentrations [4], dislocation density [1] or pinning at triple junctions [5], it is known that the displacement velocity of a GB also strongly depends on its macroscopic degrees of freedom, i.e. plane orientation and crystallographic misorientation [1, 6-10]. The understanding of this crystallographic dependency is of crucial importance as it affects the development of recrystallization textures and thus the anisotropy of the material final in-use properties. In FCC metals and particularly in Al, overwhelming data of GB migration rates are available from growth selection and bicrystals experiments. Both kind of experiments show that low angle boundaries are slower than high angle boundaries and that the misorientation of the fastest moving boundaries often correspond to a rotation around a $\langle 111\rangle$ axis with an angle close to $40^{\circ}[1,6-10]$. The two kind of experiments differ however about the nature of the main driving force for GB migration. In bicrystal experiments, the 
material is taken undeformed and thus the shrinkage of one grain should be driven only by the surface energy of its curved GB (capillary effect) [8, 10]. These experiments tend to show sharp peaks in GB mobility, e.g. a peak mobility is observed for $38.2^{\circ}\langle 111\rangle \Sigma 7$ boundaries at $430{ }^{\circ} \mathrm{C}$ and for $40.5^{\circ}\langle 111\rangle$ at $540{ }^{\circ} \mathrm{C}$ in high purity aluminium $[8,10]$. In particular, a conjecture is that special GBs with low $\Sigma$ coincidence segregate less impurities than random GBs and therefore move faster [4]. On the contrary, in growth selection experiments, the material has been previously deformed and recrystallization starts at an artificial scratch or cut $[6,7,9]$. In this case, the main driving force should be the difference of stored energy with the deformed matrix [11]. Growth selection experiments do not exhibit sharp peaks in GB velocity but rather broad peaks centered around $40^{\circ}\langle 111\rangle$ boundaries. In $\mathrm{Al}-0.05 \mathrm{wt} \% \mathrm{Si}$ for instance [9], it is reported that the highest velocities are observed for boundaries misoriented to the matrix by $\sim 30-50^{\circ}$ about axes within $\pm 10^{\circ}$ of $\langle 111\rangle$. The origin of this misorientation dependence of GB velocity is not yet fully understood and is thus poorly predicted by recrystallization models [1].

In mesoscopic models of recrystallization, GB velocity is indeed commonly expressed as $[2,3,12,13]$

$$
\boldsymbol{v}=M F \boldsymbol{N},
$$

where $N$ is the unit normal to GB and $M$ the GB mobility. Moreover, the driving force $F$ is usually computed as the sum of a positive contribution due to the difference of stored energy between the consumed and the growing grain and an opposing term due to capillarity $[3,12,13]$ :

$$
F=\eta \Delta \rho-\gamma \kappa .
$$

In (2), $\eta$ corresponds to the dislocation line energy, $\Delta \rho$ to the dislocation density difference, $\gamma$ to the GB energy and $\kappa$ to GB curvature. Within this framework, the effect of misorientation can be typically taken into account through the expression of $\gamma$, for instance by considering an extended Read-Shockley equation [13], but is generally not considered in the stored energy related to dislocations. It is however one of the purposes of the present work to show that, because of elastic anisotropy, GB misorientation can also have a significant influence on the variation of the stored energy due to dislocations.

Owing to elastic anisotropy, polycrystals have indeed heterogeneous elastic moduli [14] and image stresses are appended to the dislocation self-stress field (i.e. the one corresponding to an infinite homogeneous medium) in order to satisfy boundary conditions at interfaces. Hence, when a GB starts moving, the stress field seen by a specific dislocation changes immediately because the values of image stresses depend on the distance to GB. As a corollary, the strain energy of dislocations vary during GB motion, which is important to underline since recrystallization is driven by a reduction of the stored energy.

The image force $\boldsymbol{f}_{\boldsymbol{i m}}$ on a dislocation is the Peach-Koehler force at the dislocation position due to its stress field $\sigma$ without considering the contribution related to the infinite homogeneous medium [15-18]:

$$
\boldsymbol{f}_{i m}=(\boldsymbol{\sigma} \boldsymbol{b}) \times \boldsymbol{t},
$$

where $\boldsymbol{b}$ is the Burgers vector and $\boldsymbol{t}$ a unit vector along the dislocation line. It is worth un- 
derlying that the image force also corresponds to the negative of the gradient of the interaction energy between the dislocation and the GB $[15,17,19]$. For an infinite straight dislocation parallel to an infinite planar GB, the interaction energy remains unchanged if the dislocation is moved parallel to the planar GB. Hence, the image force is always normal to GB in this case, as shown by the expression found by Barnett and Lothe [16]:

$$
\boldsymbol{f}_{i m}=-\frac{E^{1 \infty}-E^{1-2}}{d} \boldsymbol{N} .
$$

In (4), the unit normal to GB $N$ points towards the dislocation which is in medium $1, d$ is the distance from GB, $E^{1 \infty}$ is the prelogarithmic energy factor of the same dislocation in the infinite homogenous medium 1 and $E^{1-2}$ is the prelogarithmic energy factor of the same dislocation at the interface of media 1 and 2. Equation (4) indicates that image force can either attract a dislocation towards GB if $E^{1-2}<E^{1 \infty}$, or conversely push it away if $E^{1 \infty}<E^{1-2}$, with a magnitude that increases as the inverse of the distance to GB [18, 2022]. As GB advances towards a dislocation, the value of the prelogarithmic energy factor of the dislocation will get closer and closer to $E^{1-2}$ and get further and further from $E^{1 \infty}$. As a consequence, it can be conjectured that in case of attractive image forces $\left(E^{1-2}<E^{1 \infty}\right)$, the elastic energy will decrease when GB advances towards dislocations, whereas in case of repulsive image forces $\left(E^{1 \infty}<E^{1-2}\right)$, the elastic energy will increase. Accordingly, considering the motion of a GB in presence of dislocations but over a small distance where it does not meet any of them, the migration is not only controlled by capillarity. There exists an additional effect that depends on the orientation of the Burgers vectors of the dislocations in the neighborhood and the grains misorientation. Such an effect can be energetically favorable or not. It is not taken into account by classical theories based on equation (2). The latter assume that GB migration leads to an elastic energy reduction only due to a lowering of GB energy and/or a decrease of total dislocation density as the grain with the highest dislocation density is consumed.

The present work therefore focus on the effect of crystallographic misorientation on the variation of stored energy during GB motion. The first purpose is to check the hypothesis of elastic energy decrease as GB advances towards an array of identical dislocations in case of attractive image forces and elastic energy increase in case of repulsive image forces. Then, the second objective is to quantify these changes of elastic energy. Numerical calculations of image forces and stored energy during the growth of a strain free grain within a recovered matrix containing an array of identical dislocations are therefore performed in Section 3.2. Moreover, in Section 3.3, possible correlations between the experimentally observed misorientation dependence of GB migration rate and the misorientation dependence of the image force values are explored in FCC materials. Equations needed to compute the elastic fields of a straight dislocation parallel to a planar interface in anisotropic elasticity are beforehand described in Section 2.1. A regularization procedure to compute the elastic energy is, furthermore, presented in Section 2.2. Some numerical applications are also performed in Section 3.1 in order to assess the effects of the regularization procedure on the stress fields of a dislocation in a bicrystal. All the results are finally discussed in Section 4, followed by concluding remarks in Section 5.

Throughout the paper, bold letters are used for vectors, tensors and matrices. The superscript $T$ denotes the transpose of a matrix and an overbar the conjugate of a complex function. The Einstein convention over repeated indices is used. The spatial derivative with respect to a Cartesian coordinate is indicated by a comma followed by the component index. i denotes the imaginary unit and $\Re(z)$ the real part of $z$. 


\section{Elastic fields due to a straight dislocation parallel to a planar interface}

The elastic fields of a straight dislocation parallel to a planar interface in anisotropic elastic media were thoroughly studied in the past [16, 17, 19, 23-25]. Here, an anisotropic bicrystal with a perfectly bonded interface whose normal is along the $x_{2}$ axis of a Cartesian reference frame (see Figure 1) is considered. The displacements $u_{i}$ are assumed to depend on $\left(x_{1}, x_{2}\right)$ only. The dislocation line is supposed to be along the $x_{3}$ axis and located in crystal I $\left(x_{2}>0\right)$ at the position $\left(X_{1}, X_{2}\right)$ (Figure 1). In the following, only the final equations that are needed to perform the numerical calculations are presented. More details can be found in [18, 25].

\subsection{Displacements, strains, stresses}

The values of the displacements at the point $\left(x_{1}, x_{2}\right)$ are obtained as:

$$
\begin{aligned}
u_{i} & =2 \Re\left(A_{i j} f_{j}\left(z_{j}\right)\right) \\
\text { with } f_{j}\left(z_{j}\right) & =\left\{\begin{aligned}
f_{j}^{0}\left(z_{j}\right)+f_{j}^{I}\left(z_{j}\right) & \text { if } x_{2}>0 \\
f_{j}^{I I}\left(z_{j}\right) & \text { if } x_{2}<0
\end{aligned}\right.
\end{aligned}
$$

where $z_{j}=x_{1}+p_{j} x_{2}$ and $\boldsymbol{A}$ is a $3 \times 3$ matrix containing the eigenvectors related to the eigenvalues $p_{j}$ with positive imaginary parts of the Stroh sextic equation [26, 27]:

$$
\operatorname{det}\left(C_{i 1 k 1}+p\left(C_{i 1 k 2}+C_{i 2 k 1}\right)+p^{2} C_{i 2 k 2}\right)=0 .
$$

$C$ is the elastic stiffness tensor of the medium which is considered. The function vector $f^{0}$ is related to the homogenous problem. Its general form is given by:

$$
f_{j}^{0}\left(z_{i}\right)=q_{j}^{0} \ln \left(z_{i}-s_{j}\right)
$$

where $s_{j}=X_{1}+p_{j} X_{2}$ depends on the dislocation position and $\boldsymbol{q}^{\mathbf{0}}$ is a complex vector expressed as:

$$
\begin{aligned}
\boldsymbol{q}^{\mathbf{0}} & =-\frac{1}{2 \pi} \mathrm{i}\left(\boldsymbol{B}^{T} \boldsymbol{b}\right) \\
\text { with } B_{i j} & =\left(C_{i 2 k 1}+p_{j} C_{i 2 k 2}\right) A_{k j} .
\end{aligned}
$$

Finally, the two function vectors $f^{I}$ and $f^{I I}$ can be expressed as:

$$
\begin{aligned}
f_{i}^{I}\left(z_{i}\right)=\overline{V_{i j}^{I, I I}} \overline{f_{j}^{0}}\left(z_{i}\right) & \text { if } x_{2}>0 \\
f_{i}^{I I}\left(z_{i}\right)=W_{i j}^{I, I I} f_{j}^{0}\left(z_{i}\right) & \text { if } x_{2}<0
\end{aligned}
$$

where: 


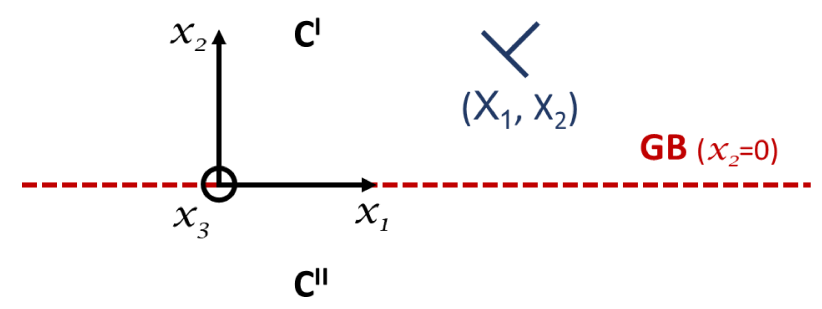

Figure 1. Straight dislocation at $\left(X_{1}, X_{2}\right)$ in an anisotropic bicrystal.

$$
\begin{aligned}
& V^{I, I I}=\left(B^{I I} A^{I I-1} \overline{A^{I}}-\overline{B^{I}}\right)^{-1}\left(B^{I}-B^{I I} A^{I I^{-1}} A^{I}\right), \\
& W^{I, I I}=\left(\overline{B^{I} A^{I^{-1}}} A^{I I}-B^{I I}\right)^{-1}\left(\overline{\left.B^{I} A^{I^{-1}} A^{I}-B^{I}\right) . ~}\right.
\end{aligned}
$$

The gradient of the displacements $u_{i, j}$ can then be expressed as:

$$
\begin{aligned}
u_{i, 1} & =2 \Re\left(A_{i j} g_{j}\left(z_{j}\right)\right) \\
u_{i, 2} & =2 \Re\left(A_{i j} p_{j} g_{j}\left(z_{j}\right)\right) \\
\text { with } g_{j}\left(z_{j}\right) & =\left\{\begin{array}{rr}
g_{j}^{0}\left(z_{j}\right)+\overline{V_{j i}^{I, I I}} \overline{g_{i}^{0}}\left(z_{j}\right) & \text { if } x_{2}>0 \\
W_{j i}^{I, I I} g_{i}^{0}\left(z_{j}\right) & \text { if } x_{2}<0
\end{array}\right. \\
\text { and } g_{i}^{0}\left(z_{j}\right) & =\frac{q_{i}^{0}}{z_{j}-s_{i}} .
\end{aligned}
$$

The strains are defined by $\varepsilon_{i j}=\frac{1}{2}\left(u_{i, j}+u_{j, i}\right)$ and the Cauchy stresses are obtained from the generalized Hooke's law: $\sigma_{i j}=C_{i j k l} \varepsilon_{k l}$.

\subsection{Elastic energy and regularization procedure}

The elastic energy per unit length on a surface area $S$ is computed by integration of the elastic energy density:

$$
w^{e}=\int_{S} \frac{1}{2} \sigma_{i j}(\boldsymbol{x}) S_{i j k l}(\boldsymbol{x}) \sigma_{k l}(\boldsymbol{x}) d^{2} \boldsymbol{x}
$$

where $\boldsymbol{S}=\boldsymbol{C}^{-1}$ is the elastic compliance tensor. The integral (12) diverges since the stress field $\sigma_{i j}(\boldsymbol{x})$ displays a singularity on the dislocation line, i.e. when $z_{j}=s_{j}$ in (11). A wellknown approach to remove this singularity in isotropic elasticity is to spread uniformly the dislocation core on the slip plane over a fixed width $2 a$ [28]. Such a procedure can be applied to the present $2 D$ configuration in anisotropic elasticity if, for instance, the dislocation is of edge character so that its Burgers vector $\boldsymbol{b}$ belongs to the plane $\left(x_{1}, x_{2}\right)$ when the dislocation line is along $x_{3}$. In this case, the intersection line of the slip plane with the plane $\left(x_{1}, x_{2}\right)$ is collinear with the Burgers vector. Hence, instead of considering a Burgers vector of magnitude $\boldsymbol{b}$ at the position $\left(X_{1}, X_{2}\right)$, a simple regularization procedure consists in spreading the 
same Burgers vector uniformly from $\left(X_{1}-a \frac{b_{1}}{b}, X_{2}-a \frac{b_{2}}{b}\right)$ to $\left(X_{1}+a \frac{b_{1}}{b}, X_{2}+a \frac{b_{2}}{b}\right)$. The latter interval has indeed a width $2 a$ and matches the slip plane trace. In this case, the displacement gradients can be obtained from (11) by replacing the function $g_{i}^{0}\left(z_{j}\right)$ as follows:

$$
\begin{aligned}
g_{i}^{0}\left(z_{j}\right) & =\int_{-a}^{a} \frac{q_{i}^{0}}{z_{j}-s_{i}} d l, \\
\text { with } s_{j} & =X_{1}+l \frac{b_{1}}{b}+p_{j}\left(X_{2}+l \frac{b_{2}}{b}\right),
\end{aligned}
$$

This procedure indeed weakens the singularity of the stress field. The singularity is now of logarithmic type and thus integrable, so that the elastic energy (12) remains finite [29].

\section{Numerical applications to $\mathrm{Al}$ and $\mathrm{Cu}$ bicrystals}

Since most of the experiments on the crystallographic dependency of GB velocities were obtained on $\mathrm{Al}[1,6-10]$, the present numerical applications are also performed on $\mathrm{Al}$, as well as on $\mathrm{Cu}$ in order to perform comparisons between two materials having dissimilar Zener anisotropy ratios $A=2 C_{44} /\left(C_{11}-C_{12}\right)$. Throughout the paper, the lattice parameters and the elastic constants of $\mathrm{Al}$ and $\mathrm{Cu}$ are considered at the same temperature of $600 \mathrm{~K}$, at which static recrystallization can occur in both materials depending of their pre-deformed state. The values are given in Table 1 . The difference of Zener ratios must be noticed, $A=1.24$ for $\mathrm{Al}$ and $A=3.40$ for $\mathrm{Cu}$. Moreover, only infinite edge dislocations are considered, both to use the regularization procedure that spreads the Burgers vector on the slip plane for the computation of elastic energy and because, within the $2 D$ Stroh formalism, screw dislocations are restricted to the very specific case of Burgers vectors parallel to GB.

\begin{tabular}{cccccc}
\hline & $a_{0}$ & $C_{11}$ & $C_{12}$ & $C_{44}$ & $A$ \\
$\mathrm{Al}$ & $4.081 \AA$ & $98 \mathrm{GPa}$ & $61 \mathrm{GPa}$ & $23 \mathrm{GPa}$ & 1.24 \\
$\mathrm{Cu}$ & $3.637 \AA$ & $158 \mathrm{GPa}$ & $118 \mathrm{GPa}$ & $68 \mathrm{GPa}$ & 3.40 \\
\hline
\end{tabular}

Table 1. Lattice parameters $\left(a_{0}\right)$ and elastic constants of $\mathrm{Al}$ and $\mathrm{Cu}$ at $600 \mathrm{~K}$ [30-32].

\subsection{Regularized dislocation stress fields in a bicrystal}

First of all, the regularization procedure proposed in (13) is checked by plotting the stress fields of an infinite straight edge dislocation in a Cu bicrystal. The configuration of section 2.1 is considered again where the normal to the planar GB is along the $x_{2}$ axis and the dislocation line vector $\boldsymbol{t}$ points positively along the $x_{3}$ axis (Figure 1). The dislocation is located in crystal $I$ at $5 b$ along its slip direction from GB. Its Burgers vectors points towards GB by making a $45^{\circ}$ angle with it. The crystallographic orientation of crystal $I$ is fixed by assuming that the dislocation belong to the slip system (111) [101]. Indeed, following the Final-Start, RightHand (FS/RH) convention, the positive normal to the slip plane is given by $\boldsymbol{n}=\boldsymbol{t} \times \boldsymbol{b} / b$. The orientation of crystal $I I$ is deduced from the one of crystal $I$ by a $40^{\circ}$ rotation around the crystallographic direction [111].

The integral in (13) is evaluated by considering that the spreading core distance is $a=1.5 b$ which is a typical value for a core radius [29], and by using a Gauss-Legendre quadrature method for which the order of the Legendre polynomial is denoted $N_{c}$. For $\mathrm{Cu}$ at $600 \mathrm{~K}$, 

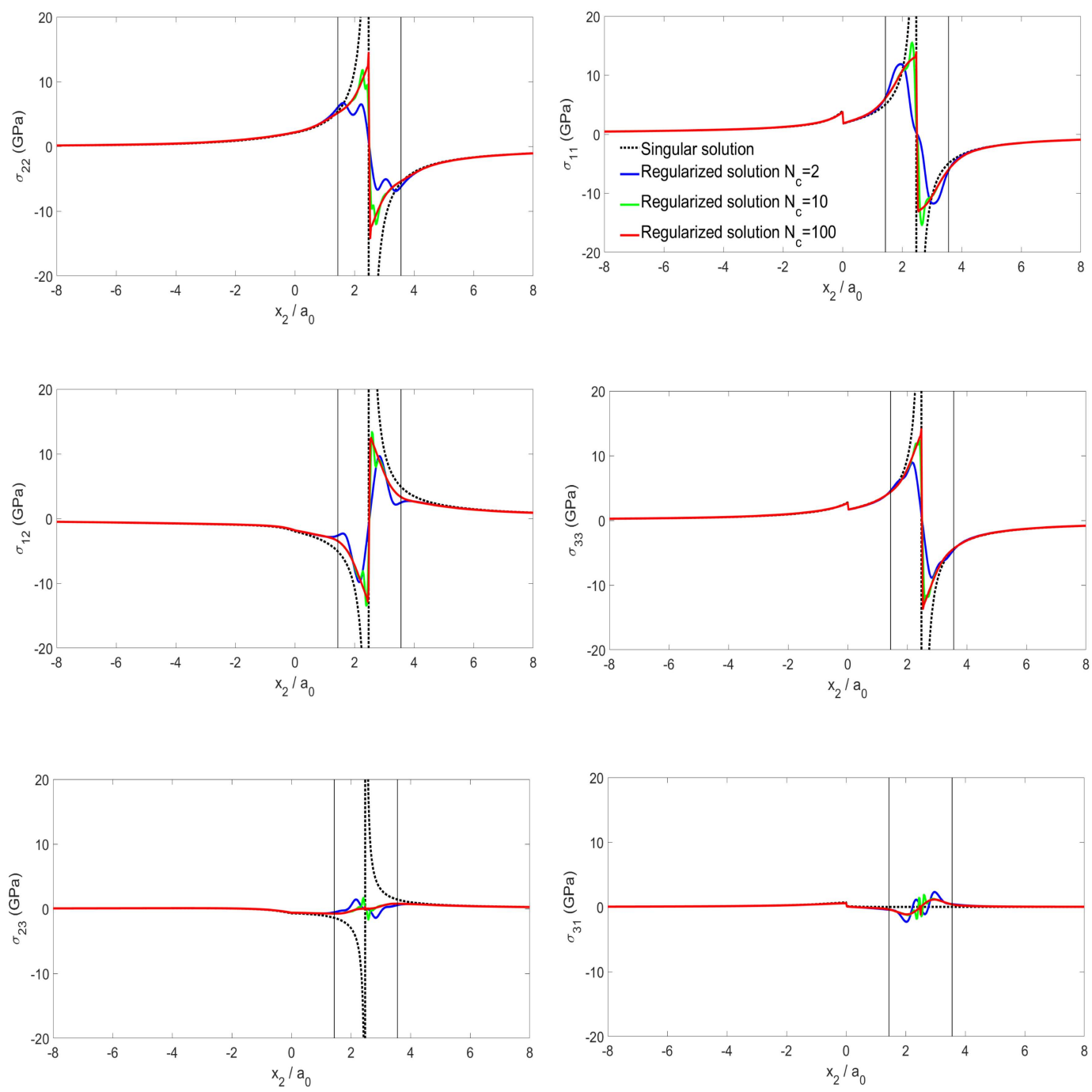

Figure 2. Variation of the six components of the Cauchy stress tensor along the $x_{2}$ axis for an infinite straight dislocation in a $\mathrm{Cu}$ bicrystal at $5 b$ along its slip direction from GB ( $\left.x_{2}=0\right)$, considering either the singular solutions (Eq. (11) in dotted lines) or the regularized ones (Eq. (13) in thick lines) with different values of $N_{c}$. The vertical lines represent the boundaries of the dislocation core considered $(a=1.5 b)$.

Figure 2 displays the variation of the six components of the Cauchy stress tensor along the $x_{2}$ axis considering either the singular solutions (11) or the regularized ones (13) with different values of $N_{c}$.

In Figure 2, it is firstly checked that the components $\sigma_{22}, \sigma_{12}$ and $\sigma_{23}$ are continuous across GB considered as a perfect interface whereas the other components display a stress jump at $x_{2}=0$. Then, it is observed that outside the dislocation core, all the regularized solutions are very close to each other and show very little departure from the singular solutions. In particular, the stress jumps are identical for all the solutions. Because the stress field is almost anti-symmetric, it is actually preferable to choose an even value of $N_{c}$ so that the singularities can compensate each other. Hence, just with $N_{c}=2$, smooth stress fields across the dislocation are obtained, which is quite satisfactory from a numerical point of view. In contrast, with $N_{c}=100$, the integral in (13) is accurately evaluated but the stress fields exhibit logarithmic singularities. 


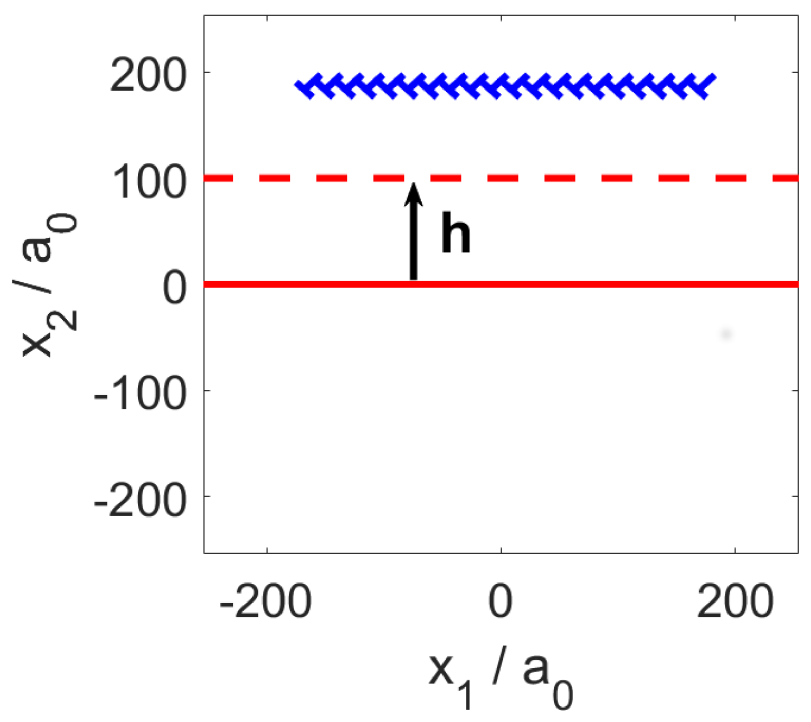

Figure 3. Array of 20 edge dislocations in an $\mathrm{Al}$ anisotropic bicrystal. The red thick line indicates the initial position of $\mathrm{GB}$ before migration starts and the red dotted line an arbitrary position during migration. $a_{0}$ is the lattice parameter. $h$ denotes the migration distance of GB.

\subsection{Elastic fields variation during grain boundary migration}

Let us consider the theoretical case of a moving planar GB that separates a grain completely free of dislocations and one that contains a finite array of 20 identical dislocations as the result of some recovery process. The configuration of section 2.1 is considered again where the normal to the planar GB is along the $x_{2}$ axis. Instead of one single dislocation, crystal $I$ now contains an array of 20 edge dislocations whose Burgers vectors belong to the $\left(x_{1}, x_{2}\right)$ plane (Figure 3).

Besides, it is assumed that the dislocations are initially in equilibrium in a zero net force configuration, as a result of the balance between the self and image stress fields of the dislocations and an internal stress field $\sigma^{i n t}$ due to defects located outside of the two grains. For the sake of simplicity, only one component of $\boldsymbol{\sigma}^{i n t}$ is assumed non-zero, the $\sigma_{22}$ one which must be continuous across the perfect interface [14]. $\sigma_{22}$ can therefore be taken homogeneous within the two grains. It is supposed that all the dislocations belong to the same slip system $(\overline{1} 11)$ [101], that the dislocation line vectors $\boldsymbol{t}$ point positively along the $x_{3}$ axis and that the Burgers vectors point towards GB and make a $45^{\circ}$ angle with it. Such a configuration is therefore consistent with an array built from dislocation glide under the main action of $\sigma^{i n t}$ and that became stable when $\sigma^{i n t}$ was balanced by the self and image stress fields of dislocations. While the crystallographic orientation of crystal $I$ is fixed following the FS/RH convention, two different orientations are considered for crystal $I I$ :

- orientation $A$ which is deduced from crystal $I$ by a $40^{\circ}$ rotation around the crystallographic direction [111],

- orientation $B$ which is deduced from crystal $I$ by a $60^{\circ}$ rotation around the crystallographic direction [110].

As it will be shown, orientation $A$ produces attractive image forces on the dislocations of crystal $I$ whereas orientation $B$ produces repulsive image forces. Therefore, in order to achieve initial equilibrium, a positive value of $\sigma_{22}^{\text {int }}$, which actually corresponds to a negative resolved shear stress, is considered for orientation $A$ and a negative one for orientation $B$. The values of $\sigma_{22}^{i n t}$ are chosen such that the initial average distances to GB are roughly similar for all the 


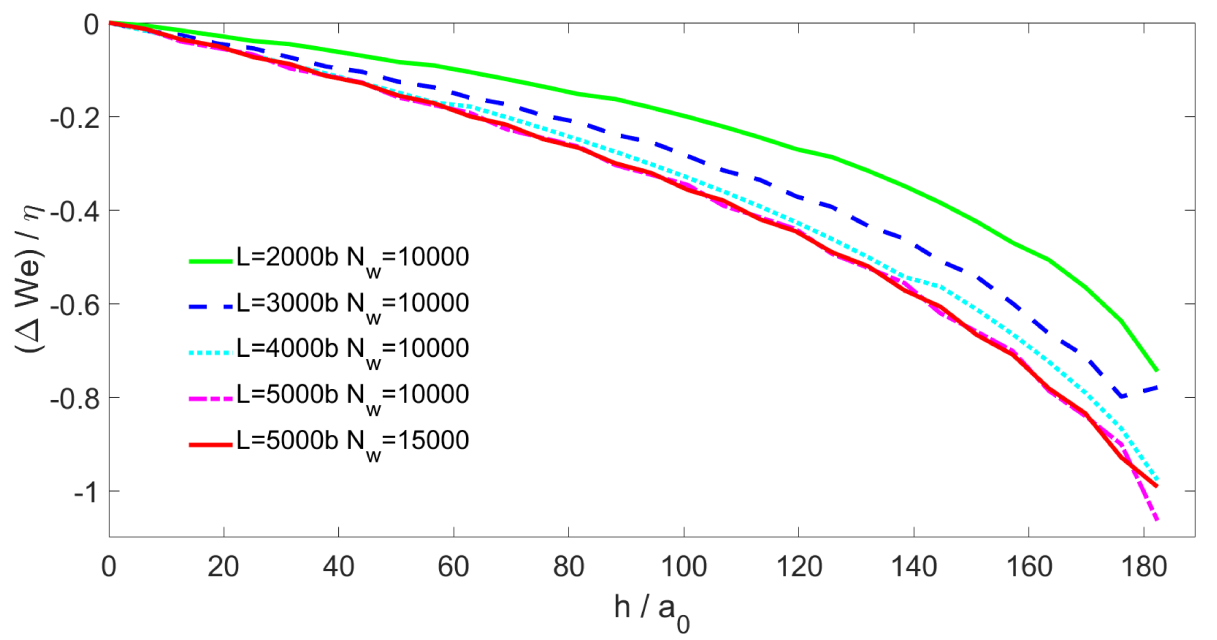

Figure 4. Variation of the stored energy from its initial value $\left(\Delta w^{e}\right)$ with the GB migration distance $(h)$ for an array of 20 dislocations in $\mathrm{Al}$ with orientation $A . \Delta w^{e}$ is normalized by the dislocation line energy $(\eta) . L$ is the parameter used to delimit the surface area of integration and $N_{w}$ the order of the Legendre polynomial used to evaluate $\Delta w^{e}$ (see text).

studied situations and are smaller than $1 \mu \mathrm{m}$. Hence, the values chosen are $\sigma_{22}^{\text {int }}=2 \mathrm{MPa}$ and $\sigma_{22}^{i n t}=-2 \mathrm{MPa}$ for orientations $A$ and $B$ in $\mathrm{Al}$ and $\sigma_{22}^{i n t}=30 \mathrm{MPa}$ and $\sigma_{22}^{i n t}=-30 \mathrm{MPa}$ for orientations $A$ and $B$ in $\mathrm{Cu}$. They correspond to initial average distances to GB of 183 , 179,177 and 162 atomic spacing $\left(a_{0}\right)$, respectively. The initial equilibrium positions of the 20 dislocations are found out by minimization of the projection of the Peach-Koehler force along the slip direction for each dislocation, which amounts to minimizing the resolved shear stress on each dislocation. In practice, a value of resolved shear stress less than $1 \mathrm{~Pa}$ is used. This minimization procedure is performed thanks to the function $f$ solve of of the software MATLAB R2019a which is based on the trust-region-dogleg numerical algorithm [33, 34]. Furthermore, these edge dislocations are restricted to remain within their slip planes which are are equally spaced along the $x_{1}$ axis by $25 b$. With this spacing distance, the misorientation induced by the dislocation array is less than $2^{\circ}$ and its effect is not taken into account in the simulation. As a result of the equilibrium procedure, the distances to GB are slightly different from one dislocation to another. The real positions are represented for orientation $A$ in $\mathrm{Al}$ in Figure 3 but the differences are hardly detectable by eye.

The local resolved shear stresses at each dislocation position as well as the stored energy per unit length are computed for different GB positions as if GB was migrating in one block towards the dislocation array. The migration is stopped when the distance between GB and the nearest dislocation is equal to $b$. Note that the positions of dislocations remain fixed and that $\sigma^{i n t}$ is also kept constant. Hence, the Peach-Koehler forces on dislocations are no more zero when the GB advances.

The elastic energy per unit length $w^{e}$ is computed by evaluating the integral (12) on a finite surface $S$ thanks to a Gauss-Legendre quadrature method and by using the regularized expression of the dislocation stress field (13) with $N_{c}=2$. It is noteworthy that the local stress field $\sigma$ is the sum of $\sigma^{i n t}$ and the self and image stress fields of all the dislocations. The orders of the Legendre polynomials to compute (12) are taken to be the same along $x_{1}$ and $x_{2}$ and denoted hereafter $N_{w}$. For a finite array of straight dislocations, $w^{e}$ depends of course on $S$. However, only the variation of $w^{e}$ from its initial value, denoted hereafter $\Delta w^{e}$, is of interest in this study. A convergence of $\Delta w^{e}$ is observed when increasing $S$ (Figure 4). Rectangular surface area are considered for $S$ with bounds corresponding to $X_{1}^{\min }-L$ and $X_{1}^{\max }+L$ along the $x_{1}$ axis and $X_{2}^{\text {min }}-L$ and $X_{2}^{\max }+L$ along the $x_{2}$ axis, $X_{1}^{\text {min }}, X_{1}^{\text {max }}, X_{2}^{\text {min }}$ and $X_{2}^{\text {max }}$ 

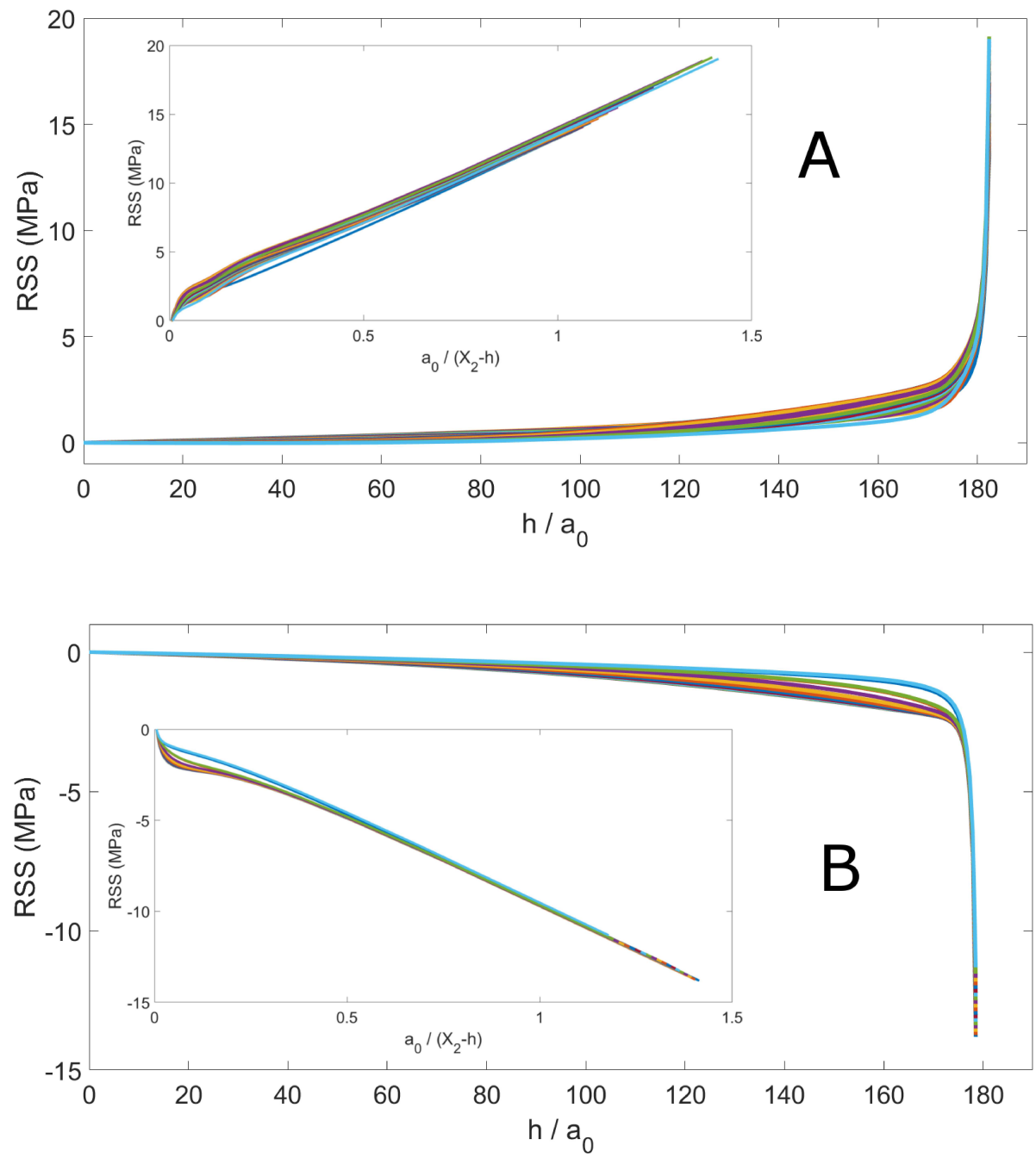

Figure 5. Variation of the resolved shear stress (RSS) on each dislocation with the GB migration distance $(h)$ for orientations $A$ and $B$ in Al. Insets: variation of the RSS on each dislocation with the inverse of the distance to GB $\left(X_{2}-h\right)$. 


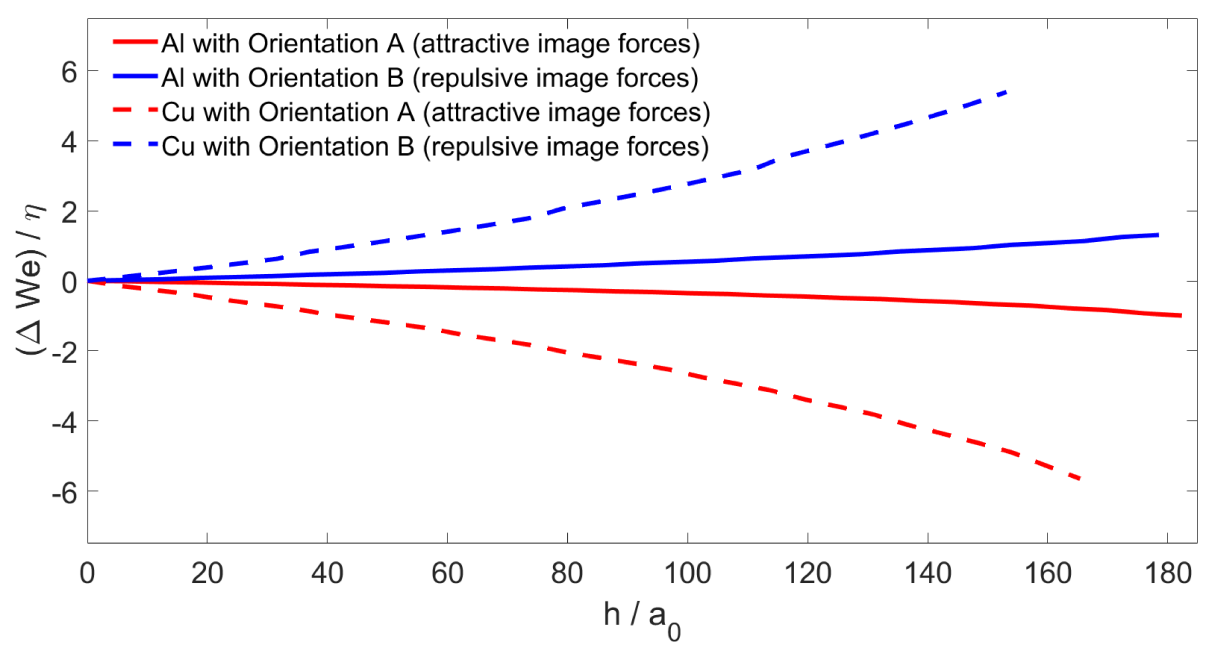

Figure 6. Variation of the stored energy from its initial value $\left(\Delta w^{e}\right)$ with the GB migration distance $(h)$ for an array of 20 dislocations in $\mathrm{Al}$ and $\mathrm{Cu}$ with orientations $A$ and $B . \Delta w^{e}$ is normalized by the dislocation line energy $(\eta)$. For all cases, $L=5000 b$ and $N_{w}=15000$.

denoting the minimum and maximum dislocation coordinates along $x_{1}$ and $x_{2}$, respectively. A good convergence is obtained for $L=5000 b$ (Figure 4). A convergence of the solutions $\Delta w^{e}$ is also reached by increasing $N_{w}$ (Figure 4). A value of $N_{w}=10000$ is necessary to obtain satisfactory results. For $L=5000 b$, the solutions obtained with $N_{w}=15000$ are very close to the ones with $N_{w}=10000$ (Figure 4), which proves that the overall trend evolution of the elastic energy is quite reliable. The variation of the stored energy is normalized by $\eta$, the dislocation line energy which is used in most mesoscopic modeling approaches to evaluate the driving force (see equation (2)). The classical approximation $\eta=\frac{1}{2} \mu b^{2}$ is considered with $\mu=20 \mathrm{GPa}$ and $\mu=45 \mathrm{GPa}$ for the isotropic shear moduli of Al and $\mathrm{Cu}$ at $600 \mathrm{~K}$, respectively.

Figure 5 exhibits the variation of the resolved shear stress (RSS) on each dislocation with the migration distance $h$ in Al. As it could be expected from equation (4), it is seen that all the RSSs roughly scale with the inverse of the distance to GB $\left(X_{2}-h\right)$ (see insets in Figure 5). Far from GB, some deviations are however noticeable due to the influence of the stress fields of other dislocations. In addition, all the RSSs are increasing for orientation $A$, i.e. image forces are attractive, whereas they are all decreasing for orientation $B$, i.e. image forces are repulsive. In parallel, it is observed that the migration of GB is accompanied by a decrease of the stored energy for orientation $A$ and an increase for orientation $B$ (Figure 6). For this array of 20 dislocations, the magnitude of $\Delta w^{e}$ is of the order of $1 \eta$ for $\mathrm{Al}$ and $6 \eta$ for $\mathrm{Cu}$ (Figure 6 ), which is significant in both cases.

In order complete the analysis, simulations are also performed for orientation $A$ in $\mathrm{Al}$ by varying the number of dislocations in the array (Figure 7) or the value of $\sigma_{22}^{\text {int }}$ (Figure 8). These variations of parameters result in different initial average distances to GB which are summarized in Tables 2 and 3. The initial average distance to GB almost scales linearly with the number of dislocations (Table 2), while for $\sigma_{22}^{i n t}$, an inverse and non-linear relation is observed (Table 3). As it could be expected, both figures indicate that the total elastic energy variation increases with the initial distance to GB. An exception is noticed however between the arrays with 10 and 15 dislocations but this is due to a sharp final drop of the the array with 10 dislocations. Indeed, it must be underlined that the variation of elastic energy near the array depends closely on the relative dislocation positions, which are fixed after initial equilibrium 


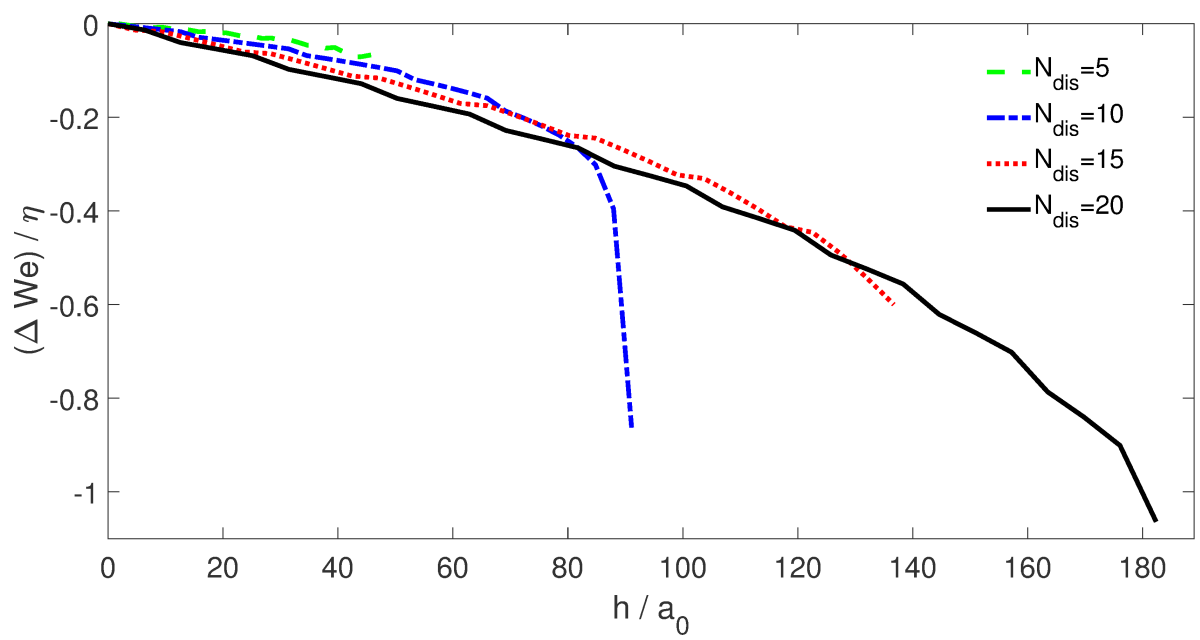

Figure 7. Variation of the stored energy from its initial value $\left(\Delta w^{e}\right)$ with the GB migration distance $(h)$ for different dislocation arrays in $\mathrm{Al}$ with orientation $A$. Different numbers of dislocations $\left(N_{\text {dis }}\right)$ are considered, resulting in different initial average distances to GB. For all cases, $\sigma_{22}^{i n t}=2 \mathrm{MPa}, L=5000 b$ and $N_{w}=10000$.

and depend on the number of dislocations. Finally, it is also shown that the magnitude of the initial gradient of elastic energy variation (i.e. the slope of $\Delta w^{e}$ vs. $h$ ) increases with both the number of dislocations (Figure 7) and the value of $\sigma_{22}^{\text {int }}$ (Figure 8). However, the dependence on $\sigma_{22}^{i n t}$ is much more pronounced.

\begin{tabular}{ccccc}
\hline$N_{\text {dis }}$ & 5 & 10 & 15 & 20 \\
Initial average distance to GB & $46 \mathrm{a}_{0}$ & $92 \mathrm{a}_{0}$ & $138 \mathrm{a}_{0}$ & $183 \mathrm{a}_{0}$ \\
\hline
\end{tabular}

Table 2. Initial average distance to GB for dislocation arrays with different numbers of dislocations $\left(N_{d i s}\right)$ in $\mathrm{Al}$ with orientation $A$ and $\sigma_{22}^{i n t}=2 \mathrm{MPa}$.

\begin{tabular}{ccccc}
\hline$\sigma_{22}^{\text {int }}$ & $1 \mathrm{MPa}$ & $2 \mathrm{MPa}$ & $3 \mathrm{MPa}$ & $4 \mathrm{MPa}$ \\
Initial average distance to GB & $505 \mathrm{a}_{0}$ & $183 \mathrm{a}_{0}$ & $83 \mathrm{a}_{0}$ & $48 \mathrm{a}_{0}$
\end{tabular}

Table 3. Initial average distance to GB for different values of $\sigma_{22}^{\text {int }}$ for an array of 20 dislocations in $\mathrm{Al}$ with orientation $A$.

\subsection{How strong are image forces at grain boundaries with $\langle 111\rangle$ misorientation axis?}

The results of the previous section show that there is a clear link between attractive image forces on dislocations and decrease of stored energy during GB motion (everything else remaining fixed) or repulsive image forces and increase of stored energy. In order to unveil GBs which are the more prone to lead to a decrease of stored energy during GB motion towards an array of identical dislocations, it is therefore conjectured that it is sufficient to compute the image force on a dislocation at a given distance from GB. Accordingly, in the following, a single straight edge dislocation parallel to GB at a distance of $5 b$ along its slip direction is always considered. The projections of the image force along the slip direction, i.e. the image resolved shear stress at the dislocation position, is computed.

First of all, twelve orientations are considered for crystal $I$ by choosing each time an edge dislocation that belongs to one of the twelve FCC slip systems (see the list of systems defined by the Schmid and Boas notations [35] in Table 4). As in the previous section, the orientations of crystal $I$ are fixed by following the FS/RH convention, setting the dislocation line along the $x_{3}$ axis and the GB normal along the $x_{2}$ axis. Within this configuration and from the image 


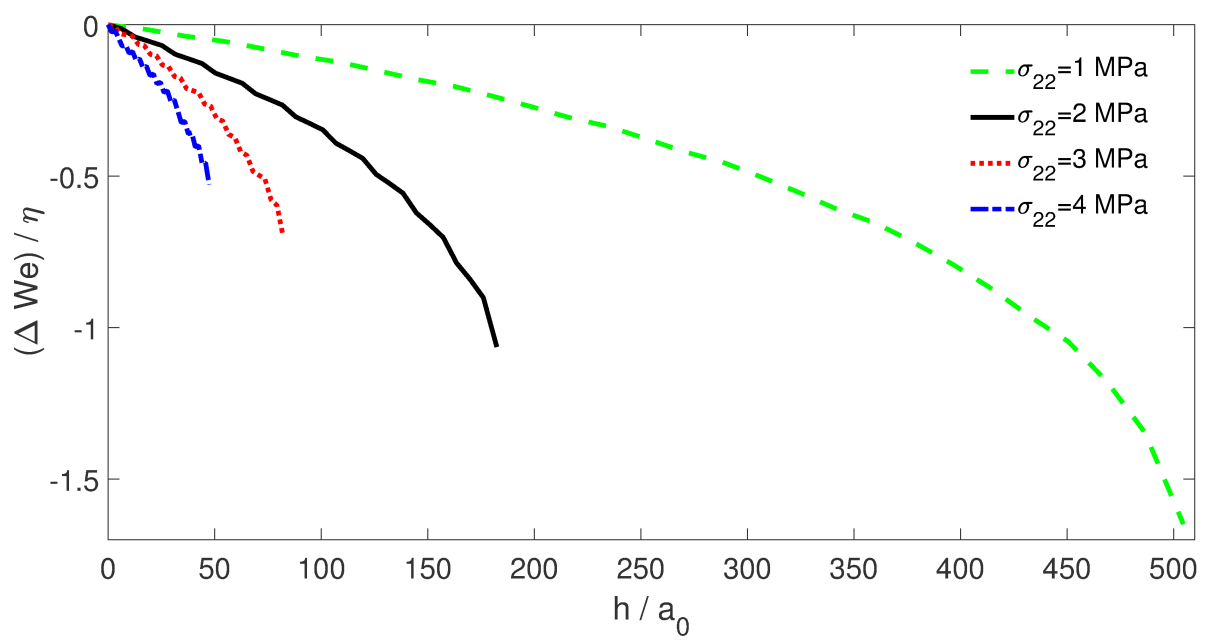

Figure 8. Variation of the stored energy from its initial value $\left(\Delta w^{e}\right)$ with the GB migration distance $(h)$ for an array of 20 dislocations in $\mathrm{Al}$ with orientation $A$. Different values of $\sigma_{22}^{i n t}$ are considered, resulting in different initial average distances to GB. For all cases, $L=5000 b$ and $N_{w}=10000$.

\begin{tabular}{|c|c|c|c|c|c|c|c|c|c|c|c|c|}
\hline Notation & A2 & A3 & A6 & B2 & B4 & B5 & $\mathrm{C} 1$ & C3 & $\mathrm{C} 5$ & D1 & D4 & D6 \\
\hline Slip planes & $(\overline{1} 11)$ & $(\overline{1} 11)$ & $(\overline{1} 11)$ & (111) & (111) & (111) & $(\overline{1} \overline{1} 1)$ & $(\overline{1} \overline{1} 1)$ & $(\overline{1} \overline{1} 1)$ & $(1 \overline{1} 1)$ & $(1 \overline{1} 1)$ & $(1 \overline{1} 1)$ \\
\hline Slip directions & {$[0 \overline{1} 1]$} & [101] & [110] & {$[0 \overline{1} 1]$} & {$[\overline{1} 01]$} & {$[\overline{1} 10]$} & {$[011]$} & {$[101]$} & {$[\overline{1} 10]$} & {$[011]$} & {$[\overline{1} 01]$} & {$[110]$} \\
\hline
\end{tabular}

Table 4. Schmid and Boas notations for slip systems in FCC crystals [35].

force theorem [16], it is known that the value of the image force is the same for all GB planes which are parallel to the dislocation line. Hence, the angle that the Burgers vector makes with GB has no influence on the image resolved shear stress and can be fixed arbitrarily. The orientations of crystal $I I$ are deduced from crystals $I$ by rotations around the specific direction [111]. It is noteworthy that the elastic stiffness tensor $C^{I}$ remains unchanged due to cubic symmetry but that $C^{I I}$ changes both with the rotation angle and the considered orientation for crystal $I$ since the rotation is always around the particular direction [111].

Figure 9 shows the variation of the image resolved shear stress (IRSS) with the misorientation angle $\theta$ for the twelve configurations, the misorientation axis being always [111]. It is observed that the three systems with a (111) plane display zero image force while three other systems ( $A 3, C 1$ and $D 6$ ) provide the exact same positive resolved shear stress on the dislocation (i.e. attractive image force) with a maximum around $38^{\circ}$. This result is intriguing regarding the measurements of GB velocities in growth selection experiments which exhibit broad peaks centered around $40^{\circ}\langle 111\rangle$ boundaries [9]. However, it must be then determined if these values of attractive image forces are significant in comparison to other possible orientations.

For this purpose, the computational methodology of Figure 9 is generalized to any kind of misorientation. In order to cover the entire orientations space, an uniform distribution of 50000 orientations in presence of cubic symmetry is generated from the open-source software package Neper [36]. This representative list of 50000 sets of Euler angles is used to define the rotations to get from crystals $I$ (twelve possible orientations) to crystals $I I .600000$ different configurations are hence studied. The results for $\mathrm{Al}$ and $\mathrm{Cu}$ are presented in Figures 10 and 11. $\langle 110\rangle,\langle 001\rangle$ and $\langle 111\rangle$ misorientation axes within $\pm 3^{\circ}$ are highlighted in Figure 10, whereas the $\langle 111\rangle$ misorientation axes within $\pm 10^{\circ}$ are highlighted in Figure 11.

It is observed that the maximum image resolved shear stress at a given misorientation angle increases with the misorientation angle and then reaches a plateau after $30^{\circ}$. The absence of symmetry between attractive and repulsive image resolved shear stresses can also be noticed. 


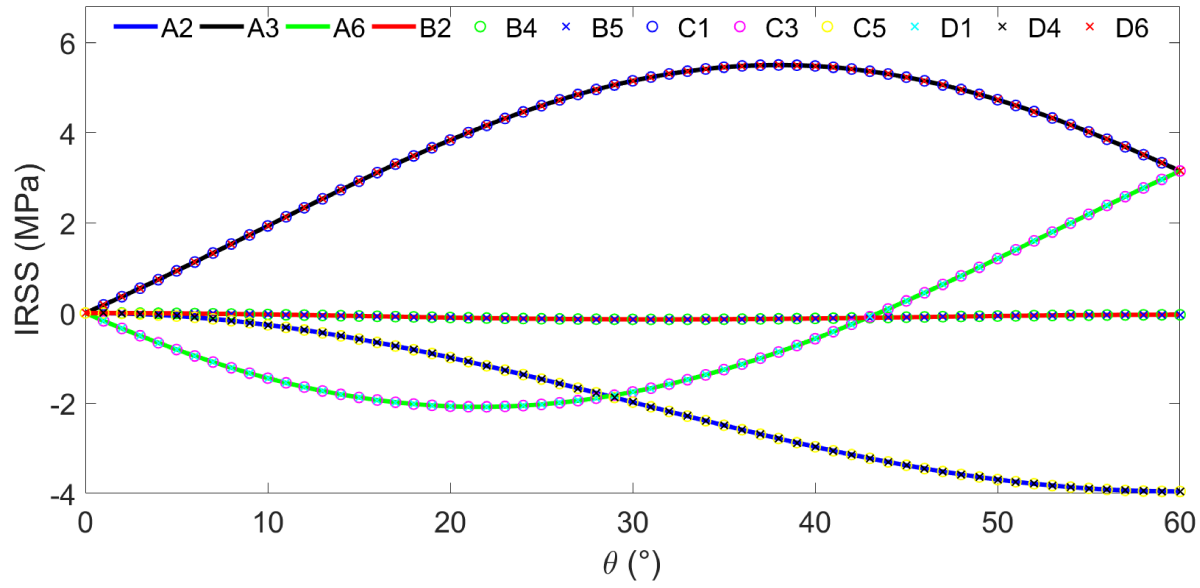

Figure 9. Variation of the image resolved shear stress (IRSS) on a straight edge dislocation in Al with the misorientation angle between grains $\theta$ for the twelve FCC slip systems when the misorientation axis is [111]. The dislocation is located at a distance of $5 b$ along its slip direction from the planar GB.
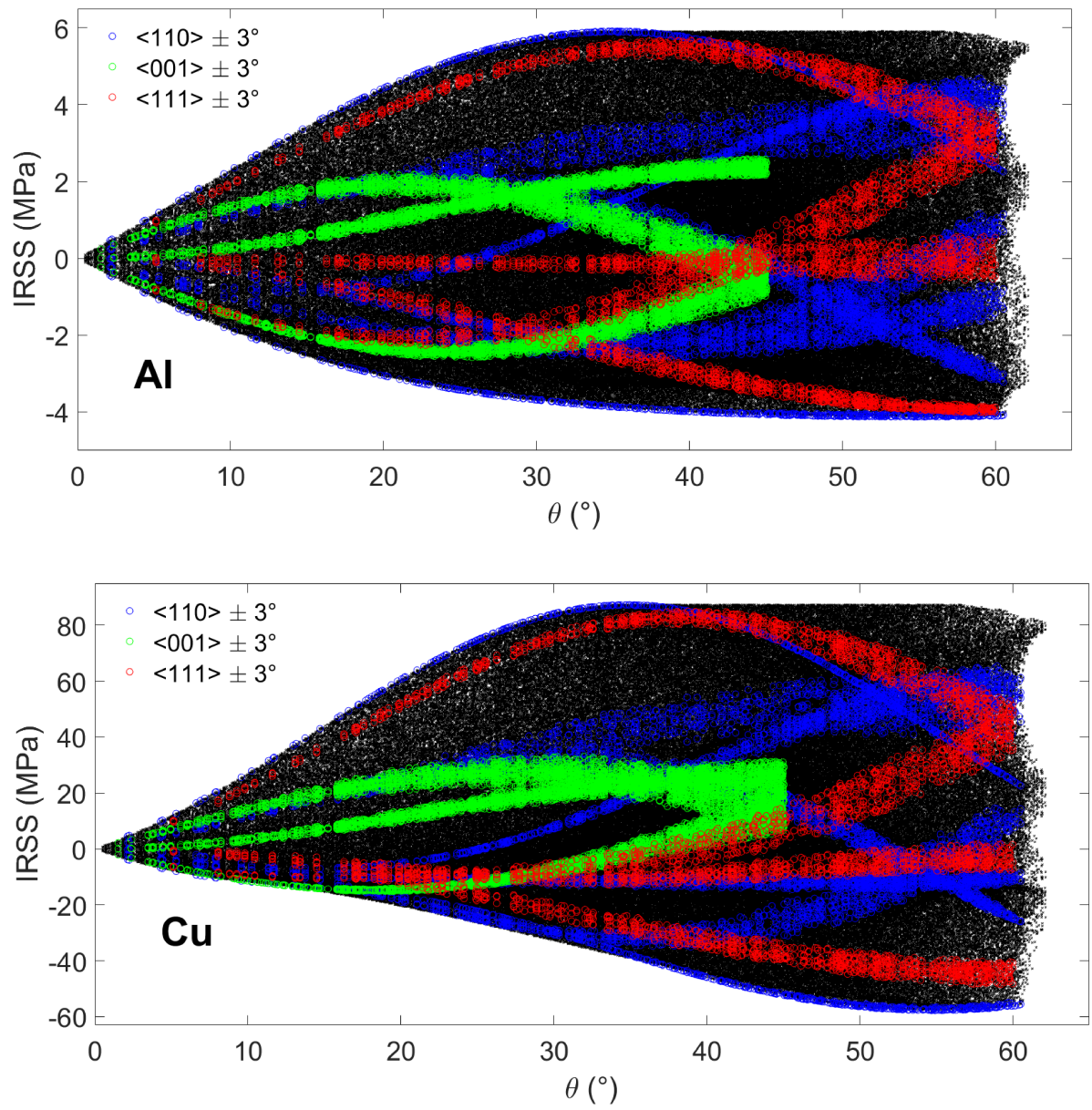

Figure 10. Clouds of points representing values of image resolved shear stresses (IRSS) on straight edge dislocations with respect to misorientation angles between grains $\theta$ for a representative set of 600000 bicrystalline configurations in $\mathrm{Al}$ and $\mathrm{Cu}$ (see text). The dislocation is located at a distance of $5 b$ along its slip direction from the planar GB. Blue, green and red circles correspond to $\langle 110\rangle,\langle 001\rangle$ and $\langle 111\rangle$ misorientation axes within $\pm 3^{\circ}$, respectively. 

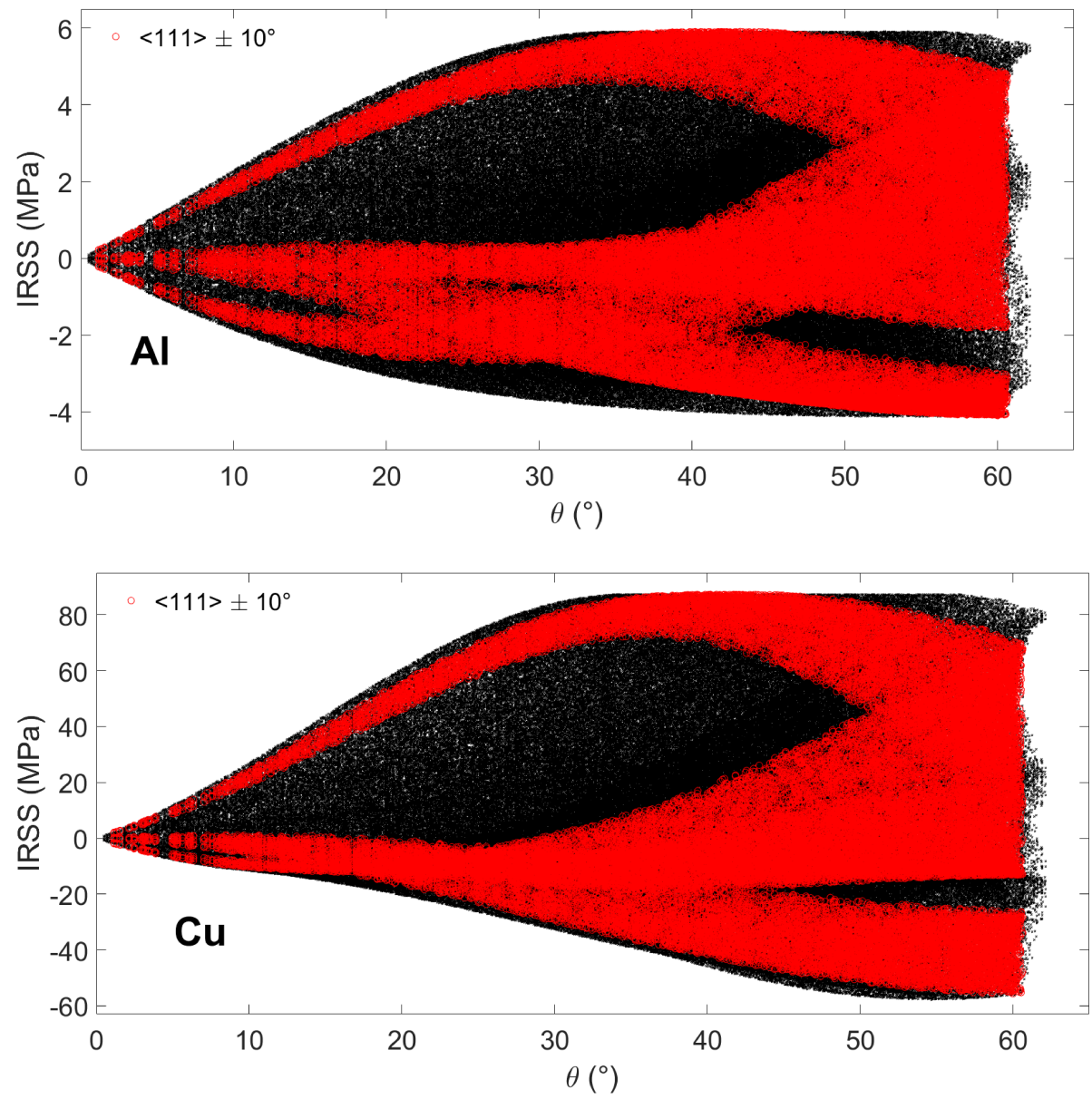

Figure 11. Clouds of points representing values of image resolved shear stresses (IRSS) on straight edge dislocations with respect to misorientation angles between grains $\theta$ for a representative set of 600000 bicrystalline configurations in $\mathrm{Al}$ and $\mathrm{Cu}$ (see text). The dislocation is located at a distance of $5 b$ along its slip direction from the planar GB. Red circles correspond to $\langle 111\rangle$ misorientation axes within $\pm 10^{\circ}$. 
Among the highest values of image resolved shear stress, there is a significant prevalence of $\langle 111\rangle$ misorientation axes within $\pm 10^{\circ}$. The frequency of the latter among the 600000 configurations is about $7 \%$ and becomes more than $13 \%$ (both in $\mathrm{Al}$ and $\mathrm{Cu}$ ) if one considers only the points having an image resolved shear stress greater than $80 \%$ of the global maximum. It is noteworthy that a reverse trend is observed for $\langle 110\rangle$ and $\langle 001\rangle$ misorientation axes. About $\langle 110\rangle \mathrm{GBs}$, it is worth underlying that they can be found both among the strongest possible repulsive image forces or among the strongest possible attractive forces for a given misorientation angle (Figure 10). This illustrates the fact that a same GB can behave very differently depending on the dislocation population in its neighborhood.

Furthermore, it is also interesting to notice that $\Sigma 3(111)$ twin boundaries, which are characterized by a $\langle 111\rangle 60^{\circ}$ misorientation and are frequent in FCC metals as annealing or mechanical twins [14], can exhibit either relatively large attractive image forces, or zero image forces or repulsive image forces very close to the highest possible values (see Figures 9 and 10). In particular, it is pointed out in the literature that the recrystallization texture of metals and alloys with a low stacking-fault energy can be significantly affected by thermal twinning and the preference of certain twin sequences [1].

\section{Discussion}

By sweeping the whole orientation space for FCC crystals, it is shown that GBs with misorientations of $\sim 30-50^{\circ}$ about axes within $\pm 10^{\circ}$ of $\langle 111\rangle$ are among those that can display the highest attractive forces on edge dislocations parallel to GB (Figure 11). From the previously developed arguments and the numerical results of subsection 3.2, it can be fairly inferred that the decrease of stored energy during migration of those GBs towards arrays of identical dislocations should lie among the strongest possible. Hence, there may be a link with the observed peaks in GB velocity centered around $40^{\circ}\langle 111\rangle$ in growth selection experiments [9].

This effect is related to elastic interactions between dislocations and GBs which are here just described as discontinuities of crystallographic orientations. More usual driving forces, like capillarity and decrease of dislocation density, are certainly predominant but elastic interactions may have non-negligible slowing-down or accelerating effects on migration. The general tendency is very clear. The migration of a GB towards dislocations on which it exerts an attraction should be accompanied by a decrease of elastic energy. On the contrary, the migration of a GB that exerts a repulsion on dislocations should be accompanied by an increase of elastic energy. As shown, this variation of elastic energy depends on crystallographic orientations but also on the dislocation slip system, as well as on the initial distance to GB, the number of dislocations and the elastic anisotropy of the material. Hence, it is important to underline that a same GB can display attractions or repulsions of different magnitudes towards a dislocation array depending on the nature of the dislocations (Figure 9). In the same way as recovery is governed by elastic interactions between dislocations, which lead to annihilation and rearrangement into ordered arrays like sub-grain boundaries, recrystallization might be controlled, at least partially, by elastic interactions between ordered arrays of dislocations and high angle grain boundaries.

Besides, the real picture is evidently much more complicated than the simplified $2 \mathrm{D}$ configurations studied in this work. In reality, dislocations are are not infinitely straight and not only of edge characters whereas GBs are not planar interfaces perfectly bonded but exhibit some curvature and imperfect bonding. In addition, elastic fields due to other defects like second phase particles, the ones due to the GB structure itself [37-40] and those due to dislocation cores were not considered in the present approach but may be taken into account 
in a future work. GBs can be modeled using ordered arrays of dislocations, disconnections or disclinations $[38,39,41]$ from which their elastic fields can be computed. GBs can also be modeled as seemingly imperfectly bonded interfaces due to the consideration of interfacial elasticity which may change the value of the image force [42]. Moreover, elastic fields of dislocation cores can be considered as well within the framework of linear heterogeneous anisotropic elasticity using force and dislocation dipoles [43, 44]. All these effects are related to the crystallography and should refine the present results.

As mentioned in the previous section, elastic rotations induced by the dislocation array were neglected in the simulations. The latter should however have some influence on the interaction with GB since a change of the misorientation angle can lead to an increase or a decrease of GB energy. Besides, it is also reminded that dislocations do not move in the present simulations, only GB does. Hence, the Peach-Koehler forces on dislocations are only zero at start and then evolve as the GB advances (Figure 5). At first, it can be assumed that these Peach-Koehler forces are below the critical force for dislocation motion and hence it is sound to consider that the dislocations remain motionless. However, it is clear that below some distance to GB (Figure 5), the dislocations should rather become mobile. In case of attractive forces for instance, dislocations should then have a 'physical interaction' with the GB (absorption, transmission, locking, etc.), which is out of the scope of the present work.

Finally, for a real polycrystalline material, an issue is to know if it is the misorientation between neighboring grains or the misorientation between the consumed grain and the mean orientation of the matrix that matters the most. This certainly depends on the distance between the dislocation array and GB: at very short distances the effect of the neighboring grain should prevail whereas at long distances the mean orientation of the matrix should have the predominant effect.

\section{Conclusions}

In this work, numerical computations of image forces and stored energy are performed in $\mathrm{Al}$ and $\mathrm{Cu}$ based on the Leknitskii-Eshelby-Stroh (LES) analytical formalism. GBs are considered as perfectly bonded planar interfaces that separate media of different crystallographic orientations. Different configurations are studied, namely the growth of a strain free grain within a recovered matrix containing a fixed array of identical edge dislocations in the case of two typical misorientations and an edge dislocation parallel to GB in the case of 600000 representative misorientations for FCC crystals. The following conclusions can be drawn:

- A regularization procedure due to Lothe [28] that extend uniformly the Burgers vector on the slip plane has been used successfully in heterogeneous anisotropic elasticity in order to evaluate the elastic energy of a dislocation array.

- The motion of a grain boundary towards a fixed dislocation array in heterogenous anisotropic elasticty can lead to an increase or a decrease of stored energy depending if the elastic interaction between the dislocation array and GB is repulsive or attractive, respectively.

- For a finite array of 20 identical edge dislocations and a GB motion over approximately 180 atomic spacing, the magnitude of the elastic energy variation is of the order of one dislocation line energy for $\mathrm{Al}$ and six for $\mathrm{Cu}$, which is significant in both cases.

- A same GB can exhibit attractions or repulsions of different magnitudes towards a dislocation array depending on the nature of the dislocations.

- In FCC crystals, GBs around a $\langle 111\rangle 40^{\circ}$ misorientation are among those that can display the highest attractive forces on edge dislocations parallel to GB, which may 
make a link with results of growth selection experiments.

\section{Funding}

The authors are grateful to the French Ministry of Higher Education and Scientific Research and the French-German University (UFA-DFH) for financial supports. The authors are also grateful to the Agence Nationale de la Recherche (ANR) for financial support under contract 'DREAM' (ANR-13-BS09-0001-03).

\section{References}

\section{References}

[1] D. Raabe, Recovery and recrystallization: Phenomena, physics, models, simulation, in Phys Metall, Laughlin, D.E., Hono, K. (Eds.), Elsevier, 2014, pp. 2291-2397.

[2] H. Stüwe and B. Ortner, Recrystallization in hot working and creep, Met Sci 8 (1974), pp. 161167.

[3] R. Ding and Z. Guo, Coupled quantitative simulation of microstructural evolution and plastic flow during dynamic recrystallization, Acta Mater 49 (2001), pp. 3163-3175.

[4] L. Shvindlerman, G. Gottstein, and D. Molodov, Grain boundary motion in pure metals: Effect of interaction between adsorbed atoms at moving boundaries, Phys Status Solidi (a) 160 (1997), pp. 419-429.

[5] B. Beucia, S. Queyreau, C. Kahloun, D. Chaubet, P. Franciosi, and B. Bacroix, Plastic straininduced grain boundary migration (sibm) in pure aluminum: Sem in-situ and afm examinations, Int J Plasticity 115 (2019), pp. 29-55.

[6] P. Beck, P. Sperry, and H. Hu, The orientation dependence of the rate of grain boundary migration, J Appl Phy 21 (1950), pp. 420-425.

[7] H. Yoshida, B. Liebmann, and K. Lücke, Orientation of recrystallized grains in strained aluminum single crystals, Acta Metall 7 (1959), pp. 51-56.

[8] D. Molodov, U. Czubayko, G. Gottstein, and L. Shvindlerman, Mobility of $<111>$ tilt grain boundaries in the vicinity of the special misorientation $\Sigma=7$ in bicrystals of pure aluminium, Scripta Metall Mater 32 (1995), pp. 529-534.

[9] Y. Huang and F. Humphreys, Measurements of grain boundary mobility during recrystallization of a single-phase aluminium alloy, Acta Mater 47 (1999), pp. 2259-2268.

[10] G. Gottstein, D. Molodov, L. Shvindlerman, D. Srolovitz, and M. Winning, Grain boundary migration: misorientation dependence, Curr Opin Solid St M 5 (2001), pp. 9-14.

[11] L. Kestens and H. Pirgazi, Texture formation in metal alloys with cubic crystal structures, Mater Sci Tech 32 (2016), pp. 1303-1315.

[12] G. Kugler and R. Turk, Modeling the dynamic recrystallization under multi-stage hot deformation, Acta Mater 52 (2004), pp. 4659-4668.

[13] M. Bernacki, H. Resk, T. Coupez, and R. Logé, Finite element model of primary recrystallization in polycrystalline aggregates using a level set framework, Model Simul Mater Sc 17 (2009), pp. 064006 (1-22).

[14] T. Richeton, I. Tiba, S. Berbenni, and O. Bouaziz, Analytical expressions of incompatibility stresses at $\Sigma 3\langle 111\rangle$ twin boundaries and consequences on single-slip promotion parallel to twin plane, Philos Mag 95 (2015), pp. 12-31.

[15] J. Eshelby, The force on an elastic singularity, Phil Trans Roy Soc London A 244 (1951), pp. $87-112$.

[16] D. Barnett and J. Lothe, An image force theorem for dislocations in anisotropic bicrystals, J Phys F Met Phys 4 (1974), pp. 1618-1635. 
[17] T. Ting and D. Barnett, Image force on line dislocations in anisotropic elastic half-spaces with a fixed boundary, Int J Solids Structures 30 (1993), pp. 313-323.

[18] X. Chen, T. Richeton, C.Motz, and S.Berbenni, Elastic fields due to dislocations in anisotropic biand tri-materials: applications to discrete dislocation pile-ups at grain boundaries, Int J Solids Structures 164 (2019), pp. 141-156.

[19] M. Tucker, Plane boundaries and straight dislocations in elastically anisotropic materials, Philos Mag 19 (1969), pp. 1141-1159.

[20] O. Khalfallah, M. Condat, and H. Kirchner, Image effects between dislocations and grain boundaries, Philos Mag A 61 (1990), pp. 291-304.

[21] . Khalfallah, M. Condat, and H. Kirchner, Image force on a lattice dislocation due to a grain boundary in b.c.c. metals, Philos Mag A 67 (1993), pp. 231-250.

[22] L. Priester and O. Khalfallah, Image force on a lattice dislocation due to a grain boundary in anisotropic f.c.c. materials, Philos Mag 69 (1994), pp. 471-484.

[23] Z. Suo, Singularities, interfaces and cracks in dissimilar anisotropic media, P R Soc London A 427 (1990), pp. 331-358.

[24] S. Choi and Y. Earmme, Elastic study on singularities interacting with in- terfaces using alternating technique, Int J Solids Structures 39 (2002), pp. 943-957.

[25] C. Hwu, Anisotropic Elastic Plates, Springer, US, 2010.

[26] A. Stroh, Dislocations and cracks in anisotropic elasticity, Philos Mag 3 (1958), pp. 625-646.

[27] A. Stroh, Steady state problems in anisotropic elasticity, J Math Phys 41 (1958), pp. 77-103.

[28] J. Lothe, Dislocations in continuous elastic media, in Elastic Strain Fields and Dislocation Mobility, Indenbohm, V.L., Lothe, J. (Eds.), Amsterdam, North-Holland, 1992, pp. 175-236.

[29] W. Cai, A. Arsenlis, C. Weinberger, and V. Bulatov, A non-singular continuum theory of dislocations, J Mech Phys Solids 54 (2006), pp. 561-587.

[30] H. Ledbetter and E. Naimon, Elastic properties of metals and alloys. ii. copper, J Phys Chem Ref Data 3 (1974), pp. 897-935.

[31] J. Tallon and A. Wolfenden, Temperature dependence of the elastic constants of aluminium, J Phys Chem Solids 40 (1979), pp. 831-837.

[32] R. Hearmon, Temperature dependence of the elastic constants of aluminium, Solid State Commun 37 (1981), pp. 915-918.

[33] J. Moré and D. Sorensen, Computing a trust region step, SIAM J Sci Stat Comp 3 (1983), pp. 553-572.

[34] M. Powell, A fortran subroutine for solving systems of nonlinear algebraic equations, in Numerical Methods for Nonlinear Algebraic Equations, chap. 7, P. Rabinowitz, ed., 1970.

[35] E. Schmid and W. Boas, Kristallplasticität, Springer Verlag, Berlin, 1935.

[36] R. Quey, A. Villani, and C. Maurice, Nearly uniform sampling of crystal orientations, J Appl Crystallogr 51 (2018), pp. 1162-1173.

[37] R. Dingreville and J. Qu, Interfacial excess energy, excess stress and excess strain in elastic solids: Planar interfaces, J Mech Phys Solids 56 (2008), pp. 1944-1954.

[38] A. Vattré and M. Demkowicz, Determining the Burgers vectors and elastic strain energies of interface dislocation arrays using anisotropic elasticity theory, Acta Mater 61 (2013), pp. 5172 5187.

[39] V. Taupin, L. Capolungo, C. Fressengeas, A. Das, and M. Upadhyay, Grain boundary modeling using an elasto-plastic theory of dislocation and disclination fields, J Mech Phys Solids 61 (2013), pp. 370-384.

[40] R. Dingreville and S. Berbenni, On the interaction of solutes with grain boundaries, Acta Mater 104 (2016), pp. 237-249.

[41] J. Hirth and R. Pond, Steps, dislocations and disconnections as interface defects relating to structure and phase transformations, Acta Mater 44 (1996), pp. 4749-4763.

[42] P. Juan and R. Dingreville, Elastic greens function in anisotropic bimaterials considering interfacial elasticity, J Elast 131 (2018), pp. 277-296.

[43] P. Gehlen, J. Hirth, R. Hoagland, and M. Kanninen, A new representation of the strain field associated with the cube-edge dislocation in a model of a $\alpha$-iron, J Appl Phys 43 (1972), pp. 3921-3933. 
[44] E. Clouet, Dislocation core field. I. Modeling in anisotropic linear elasticity theory, Phys Rev 84 (2011), pp. $224111(1-7)$. 